Research Article

\title{
Analog Circuit Soft Fault Diagnosis Based on Sparse Random Projections and K-Nearest Neighbor
}

\author{
Jian Sun $\mathbb{D}^{1}{ }^{1}$ Guobin Hu, ${ }^{1}$ and Chenghua Wang ${ }^{2}$ \\ ${ }^{1}$ College of Electronic and Information Engineering, Jinling Institute of Technology, Nanjing 211169, China \\ ${ }^{2}$ College of Electronic and Information Engineering, Nanjing University of Aeronautics and Astronautics, Nanjing 210016, China
}

Correspondence should be addressed to Jian Sun; sunjian2016@jit.edu.cn

Received 19 August 2021; Accepted 21 October 2021; Published 2 November 2021

Academic Editor: Zeeshan Pervez

Copyright $@ 2021$ Jian Sun et al. This is an open access article distributed under the Creative Commons Attribution License, which permits unrestricted use, distribution, and reproduction in any medium, provided the original work is properly cited.

Analog circuit fault diagnosis is a key problem in theory of circuit networks and has been investigated by many researchers in recent years. An approach based on sparse random projections (SRPs) and K-nearest neighbor (KNN) to the realization of analog circuit soft fault diagnosis has been presented in this paper. The proposed method uses the wavelet packet energy spectrum and sparse random projections to preprocess the time response for feature extraction. Then, the variables of the fault features are constructed, which are used to form the observation sequences of K-nearest neighbor classifier. K-nearest neighbor classifier is used to accomplish the fault diagnosis of analog circuit. In this paper, four-opamp biquad high-pass filter has been used as simulation example to verify the effectiveness of the proposed method. The simulations show that the proposed method offers higher correct fault location rate in analog circuit soft fault diagnosis application as compared with the other methods.

\section{Introduction}

With the rapid development of the microelectronics industry, fault diagnosis of mixed-signal integrated circuits is an essential area of scientific research [1-5]. At present, there are well-consolidated fault diagnosis techniques for digital circuits. However, fault diagnosis of analog circuits is still a challenging and complex task due to the lack of the simple fault model, the presence of component tolerance, the disturbance of noise, and the influence of nonlinear property $[6,7]$. In recent years, a number of scientists are getting more attention in the fields of analog circuit fault diagnosis, and many methods have been appeared [8-12].

The techniques used in the analog circuit fault diagnosis can be divided into two broad categories: the parameter estimation methods and pattern recognition methods. The parameter estimation methods require complex mathematical models. Thus, the application of parameter estimation methods is very limited in practice [13]. Recently, pattern recognition methods have been widely used in analog circuit fault diagnosis. Fault feature extraction and fault classification are two critical processes for analog circuit fault diagnosis in pattern recognition methods. Through fault feature extraction, dimensions of fault feature can be reduced and redundancies of fault feature can be removed. It would be convenient to recognize fault category of analog circuit [14-17]. It is common to see that signal processing methods, information entropy methods, frequency analysis methods, and time-domain analysis methods are used to extract fault feature in analog circuit fault diagnosis [18]. In addition, principle component analysis (PCA) and linear discriminant analysis (LDA) are used to reduce the dimensionality of fault feature in analog circuit fault diagnosis $[19,20]$. On the other hand, to design a robust classifier is also of the key importance for analog circuit fault diagnosis. Some machine learning algorithms are widely applied to diagnosis analog circuit fault, such as artificial neural network (ANN), support vector machine (SVM), support vector data description (SVDD), and Bayes network and fuzzy theory [21-23]. However, the network structure, weight, and threshold of artificial neural network are difficult to determine, and artificial neural network suffers from low convergence speed in fault diagnosis, which lead to be a poor efficiency in analog circuit fault diagnosis based on artificial neural network. Penalty parameter and kernel parameter influence SVM performance. It is difficult 
to determine kernel functions of SVM $[24,25]$. These affect the performance in analog circuit fault diagnosis.

$\mathrm{K}$-nearest neighbor is a machine learning algorithm based on statistical characteristic. It classifies the test sample throughout estimating the distances of different eigenvalues and has the advantage of simple structure, no need for training and estimation parameter. The KNN algorithm has a good classification effect on overlap data. Thus, it is appropriate to use KNN classifier for analog circuit fault diagnosis. It has been recognized that fault diagnosis based on KNN can effectively deal with some characteristics of analog circuit, such as component tolerance and nonlinearity [26]. However, the computation complexity and storage space involved in neighbors searching of KNN prevent it from online monitoring, especially for multiple faults and high-dimensional cases [27]. One possible solution to this problem is to reduce the dimensionality of the data, ideally without losing predictive performance. The reason why KNN can deal with the problems in analog circuit fault is that the distances between local samples are utilized to perform fault detection. Therefore, the dimension reduction cannot cause the distance distortion and must guarantee the performance of $\mathrm{KNN}$ in the subspace. Random projections are a powerful method of dimensionality reduction that preserves the structure of the data without introducing significant distortion. The similarity of data vectors is preserved well under random projections. Random projections do not need to decompose matrix, so it have lower computational requirement. Sparse random projections can further improve computational efficiency, and it is extremely suitable for applications of high dimension and limited samples. The advantages of SRP make it be very suitable for analog circuit fault diagnosis [28, 29]. A fault diagnosis method combining random projection and K-nearest neighbor rule can realize reducing computational complexity while guaranteeing the performance of fault detection.

To improve efficiency of analog circuit fault diagnosis, a new approach is introduced to diagnose fault via sparse random projection and K-nearest neighbor classifier for analog circuit in this paper. The proposed method not only can reduce the computational complexity and storage space but also approximately guarantee the advantages of KNN classifier in dealing with the problems of component tolerance and nonlinearity that often exist in analog circuit.

\section{Materials and Methods}

2.1. Sparse Random Projections. Random projections are an approximate algorithm for estimating distances between pairs of points in a high-dimensional vector space. It uses a suitable scale random matrix whose columns have unit lengths to project the high-dimensional feature vector down to a much lower-dimensional subspace, thus achieving the dimensional reduction of the original data set. Random projections are extremely suitable for applications of high dimension and limited samples [30]. Recently, it has been extensively applied in several areas, such as machine learning, image processing, and compressed sensing. The random projections algorithm is implemented as follows.
Let $X_{d \times n}$ be an original high-dimensional matrix, where $d$ is the dimensionality. Let $R_{k \times d}$ be random projection matrix, where $k \leq d$. The transformation of dimensionality reduction is given by the following equation:

$$
X_{k \times N}^{R P}=R_{k \times d} X_{d \times N}
$$

where $X_{k \times N}^{R P}$ is a low-dimensional matrix obtained after random projections. Random projections reduce data dimension, while approximately preserving the distance between pairs of mapping points in the Euclidean space. The data vectors in the low-dimensional matrix have important characteristic information of original data vectors. Therefore, random projections preserve the similarities of data vectors to a high degree. This theory can be derived from the famous JL (Johnson-Lindenstrauss) theorem. JL theorem is defined as follows:

$$
k \geq 4\left(\frac{\varepsilon^{2}}{2}-\frac{\varepsilon^{3}}{3}\right)^{-k} \ln n
$$

where $W$ is an arbitrary dataset in d-dimensional Euclidean space. There is a map $f: R^{d} \longrightarrow R^{k}$, and any two data $u$ and $v$ in set $W$ satisfy the following formula:

$$
(1-\varepsilon)\left\|(u-v)^{2}\right\| \leq\|f(u)-f(v)\|^{2} \leq(1+\varepsilon)\|u-v\|^{2} .
$$

JL theorem indicates that $n$ data in high-dimensional Euclidean space can map into $O\left(\log n / \varepsilon^{2}\right)$ dimensional subspace, and the distance between these data after projection is still approximate. The change distance is within $1 \pm \varepsilon$ disturbance bound.

The above analysis shows that the key to achieving data dimensionality reduction using the random projections method is to determine the appropriate random projections matrix. At present, the Gaussian random matrix is commonly used. Each of its elements is independent and subject to standard normal distribution. However, the Gaussian random matrix is more complicated for constructing largescale random matrices and has a higher computational complexity for the projection operation of data. Bingham et al. [31] proposed that two simple distributions produce a random projections matrix. The elements are independent of each other in the Gaussian random matrix. Their values will be present in the form as follows:

$$
\begin{aligned}
& r_{i, j}=\left\{\begin{array}{ll}
+1, & P=\frac{1}{2} \\
-1, & P=\frac{1}{2}
\end{array},\right. \\
& r_{i, j}= \begin{cases}+1, & \mathrm{P}=\frac{1}{6} \\
0 & \mathrm{P}=\frac{2}{3} . \\
-1, & \mathrm{P}=\frac{1}{6}\end{cases}
\end{aligned}
$$


The above two distributions greatly reduce the computational complexity of random projections matrix mapping conversion. Especially in equation (5), it can be seen from the construction that the matrix is very sparse. The elements in matrix have values of 0 with probability $2 / 3$, and $2 / 3$ projection calculated amount can be saved. So, the operation is faster. The sparse random projections matrix is proposed in this paper.

2.2. K-Nearest Neighbor. KNN is a supervised classifier. This algorithm calculates the similarity between the data samples and all neighbors and finds the $k$ most similar samples. This algorithm classifies a new entity based on decision [32]. The time required may be significantly reduced by using the dimensionality reduction while still maintaining a high accuracy in KNN. Assume that the fault sample to be diagnosed is $X_{s}$. Given training data set $U=\left\{\left(x_{i}, c_{i}\right) \mid i=1,2, \cdots, N\right\}$, which contains $m$ categories. The category set is $C=\left\{c_{l} \mid l=1,2, \cdots, m\right\}$, where training sample $x_{i}$ is a p-dimensional column vector and $c_{i}$ is the category label corresponding to $x_{i}$. Steps in KNN classifier algorithms are as follows:

(1) Calculate the distance between the samples $X_{s}$ to be classified and all training samples. Euclidean distance, Manhattan distance, and Minkowski distance are usually used as the distance measured between the test object and every object in the set of training objects. Euclidean distance function is used in this paper.

$$
d\left(x_{s}, x_{i}\right)=\sqrt{\left(x_{s}-x_{i}\right)^{T}\left(x_{s}-x_{i}\right)} .
$$

(2) Select nearest neighbor parameter $k$. Find K-nearest neighbor samples $X_{s}$ according to the calculated Euclidean distance, and it is written as follows: $Q_{s}=\left\{\left(x_{s j}, c_{s j}\right) \mid j=1,2, \cdots, k\right\}$, where $x_{s j}$ is the jth $(1 \leq j \leq k)$ nearest neighbor sample of the sample $X_{s}$ to be classified and $c_{s j}$ is the category corresponding to $x_{s j}$.

(3) Vote based on the category information of the $\mathrm{K}$-nearest neighbor samples in $Q_{s}$. The result of the vote is written as follows: $V=\left[v_{1}, v_{2}, \cdots, v_{l}, \cdots v_{M}\right]$. To make decisions according to the voting results, the decision rule is as follows:

$$
c\left(x_{s}\right)=\arg \max \left(v_{l}\right) .
$$

\section{Analog Circuit Fault Diagnosis Model Based on SRP-KNN}

Use four-opamp biquad high-pass filter circuit shown in Figure 1 as example to introduce the analog circuit soft fault diagnosis method based on SRP-KNN. There are four opamps, two capacitors, and ten resistors in four-opamp biquad high-pass filter circuit. Capacitors and resistors are used as fault components to diagnose.

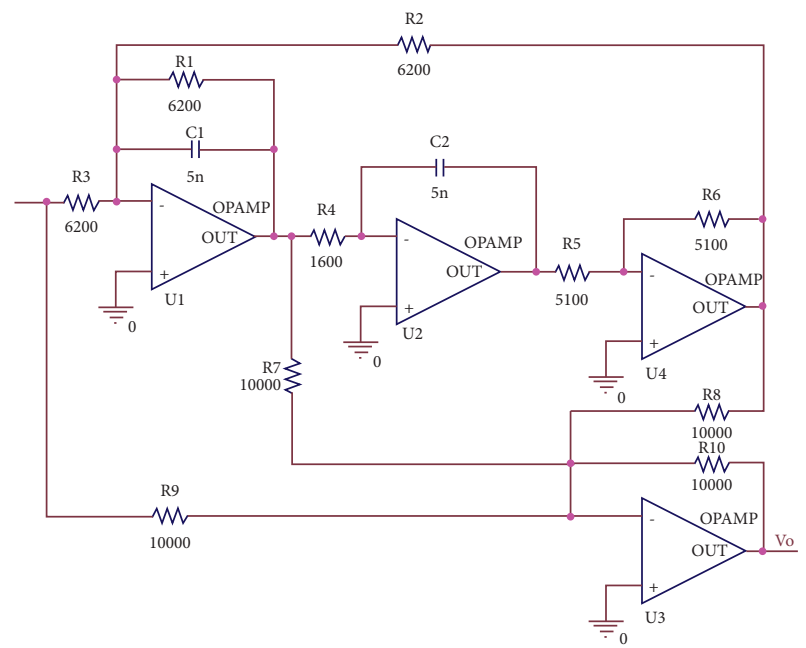

FIgURE 1: Four-opamp biquad high-pass filter circuit.

Figure 2 shows four-opamp biquad high-pass filter amplitude-frequency response of different fault modes stimulated by a $1 \mathrm{~V}$ AC signal. It can be seen from Figure 2 that when a component of the circuit fails, its amplitudefrequency response curve will change. Therefore, the fault characteristics of the analog circuit can be acquired through the response curve.

The flow chart of analog circuit fault diagnosis based on SRP-KNN is shown in Figure 3. In the first place, input a drive signal to the analog circuit under test. Simultaneously, collect wavelet packet energy spectrum fault features at the output of the circuit. Using the sparse random projections algorithm to reduce the dimensions of the collected fault feature, normalization processing is applied to these data. The samples are divided into train samples and test samples. The results of analog circuit fault diagnosis are obtained by KNN classifier.

\section{Experiment Results and Analysis of Simulation}

The example for analog circuit is a four-opamp biquad highpass filter, which is shown as Figure 1. It consists of 4 operational amplifiers, 2 capacitors, and 10 resistors. The nominal value of each component is indicated in Figure 1 wherein the resistor and capacitor each has a tolerance of $1 \%$. If the values of resistor and capacitor are outside the tolerance range and are within 50\%, then the circuit is considered to have a soft fault.

Select $\mathrm{C}_{1}, \mathrm{C}_{2}, R_{1}, R_{2}, R_{3}$, and $R_{4}$ as diagnostic components by sensitivity analysis. The soft fault of each component has two modes: a large soft fault and a small soft fault. Use $\uparrow$ to indicate that the component has a large fault and $\downarrow$ to indicate that the component has a small fault. There are $13 \mathrm{soft}$ fault modes including fault-free state (NF), which are listed in Table 1.

OrCAD10.5 software is used to produce the simulation fault data according to the fault modes in Table 1 . The circuit is inputted a pulse stimulus with the amplitude of $5 \mathrm{~V}$ and 


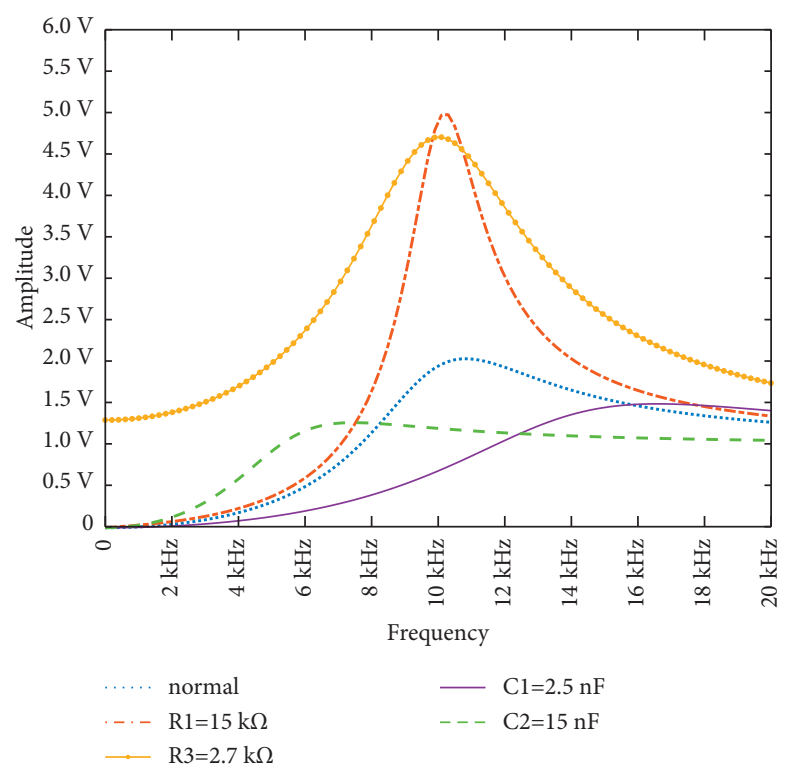

FIGURE 2: Amplitude-frequency response curve of four-opamp biquad high-pass filter.

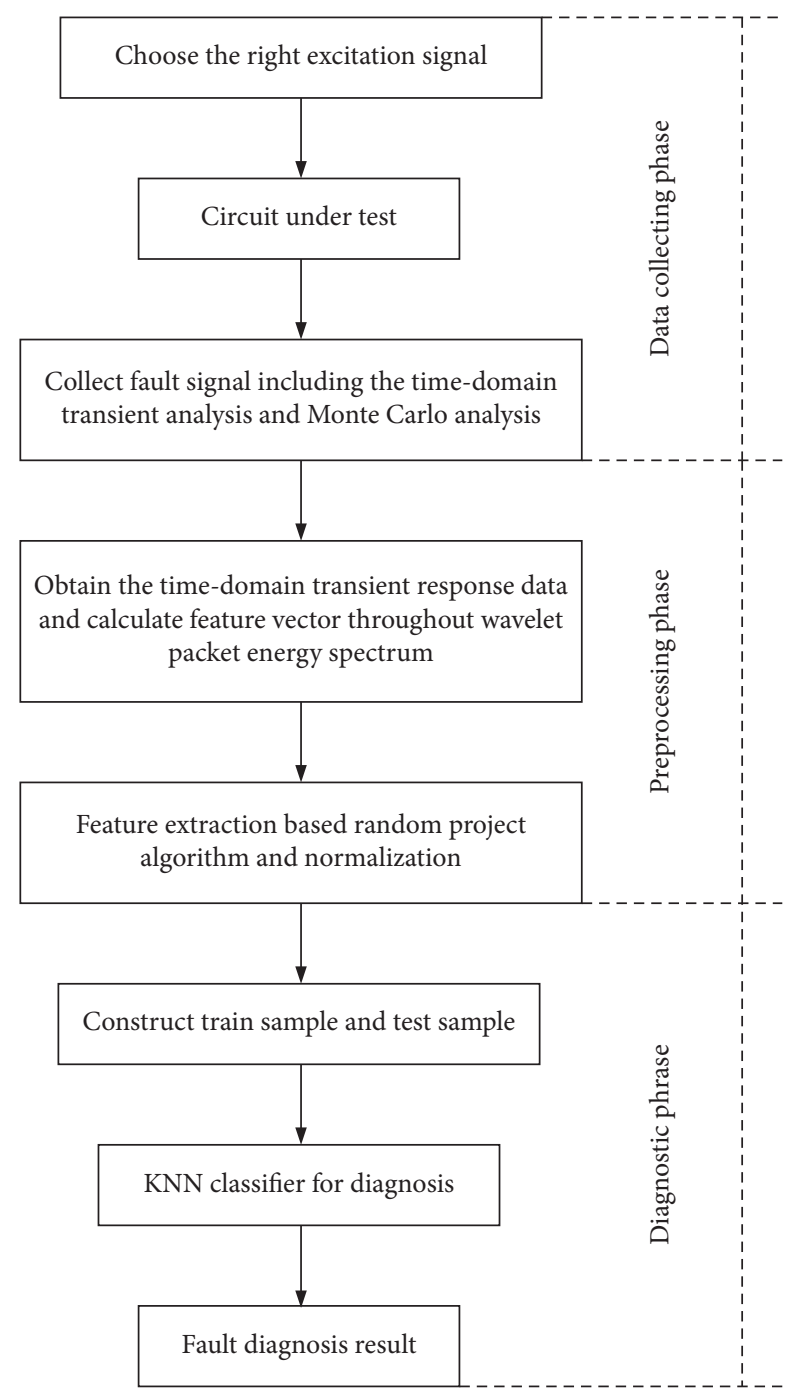

FIGURE 3: Flow chart of analog circuit fault diagnosis based on SRPKNN.
TABLE 1: Soft fault modes of four-opamp biquad high-pass filter.

\begin{tabular}{lccc}
\hline Fault code & Fault model & Nominal value & Fault value \\
\hline$F 1$ & $C 1 \uparrow$ & $5 \mathrm{nF}$ & $6 \mathrm{nF}$ \\
$F 2$ & $C 1 \downarrow$ & $5 \mathrm{nF}$ & $4 \mathrm{nF}$ \\
$F 3$ & $C 2 \uparrow$ & $5 \mathrm{nF}$ & $6 \mathrm{nF}$ \\
$F 4$ & $C 2 \downarrow$ & $5 \mathrm{nF}$ & $4 \mathrm{nF}$ \\
$F 5$ & $R 1 \uparrow$ & $6.2 \mathrm{k} \Omega$ & $7.5 \mathrm{k} \Omega$ \\
$F 6$ & $R 1 \downarrow$ & $6.2 \mathrm{k} \Omega$ & $5 \mathrm{k} \Omega$ \\
$F 7$ & $R 2 \uparrow$ & $6.2 \mathrm{k} \Omega$ & $7.5 \mathrm{k} \Omega$ \\
$F 8$ & $R 2 \downarrow$ & $6.2 \mathrm{k} \Omega$ & $5 \mathrm{k} \Omega$ \\
$F 9$ & $R 3 \uparrow$ & $6.2 \mathrm{k} \Omega$ & $7.5 \mathrm{k} \Omega$ \\
$F 10$ & $R 3 \downarrow$ & $6.2 \mathrm{k} \Omega$ & $5 \mathrm{k} \Omega$ \\
$F 11$ & $R 3 \uparrow$ & $1.6 \mathrm{k} \Omega$ & $2 \mathrm{k} \Omega$ \\
$F 12$ & $R 4 \downarrow$ & $1.6 \mathrm{k} \Omega$ & $1.2 \mathrm{k} \Omega$ \\
$F 13$ & $\mathrm{NF}$ & -- & -- \\
\hline
\end{tabular}

pulse width of 10 us and implemented time-domain transient analysis and 50 Monte Carlo analyses. In the timedomain transient analysis, the run-to-time and maximum step size are set as $0.5 \mathrm{~ms}$ and 4 us. In the Monte Carlo analysis, the number of runs is set to 50 and distribution is set to uniform distribution. There are 50 time-domain response curves for each fault mode (including fault-free). In each time-domain transient response curve, there are 125 points. The time-domain transient fault response curves of four-opamp biquad high-pass filter (C1 big fault, $\mathrm{C} 2$ small fault, and normal state) using the time-domain transient analysis and the Monte Carlo analysis are shown in Figure 4.

Then, three-layer Haar wavelet packet decomposition is performed on the collected fault signal. Eight bands wavelet packet reconstruction signals of the third layer are obtained, respectively. The energy spectrums in its eight bands are calculated, and the energy spectrum values are taken as fault features. Fault curves of 3-layer Haar wavelet packet decomposition ( $C_{1}$ big fault, $C_{2}$ small fault, and normal state) are drawn in Figure 5. 

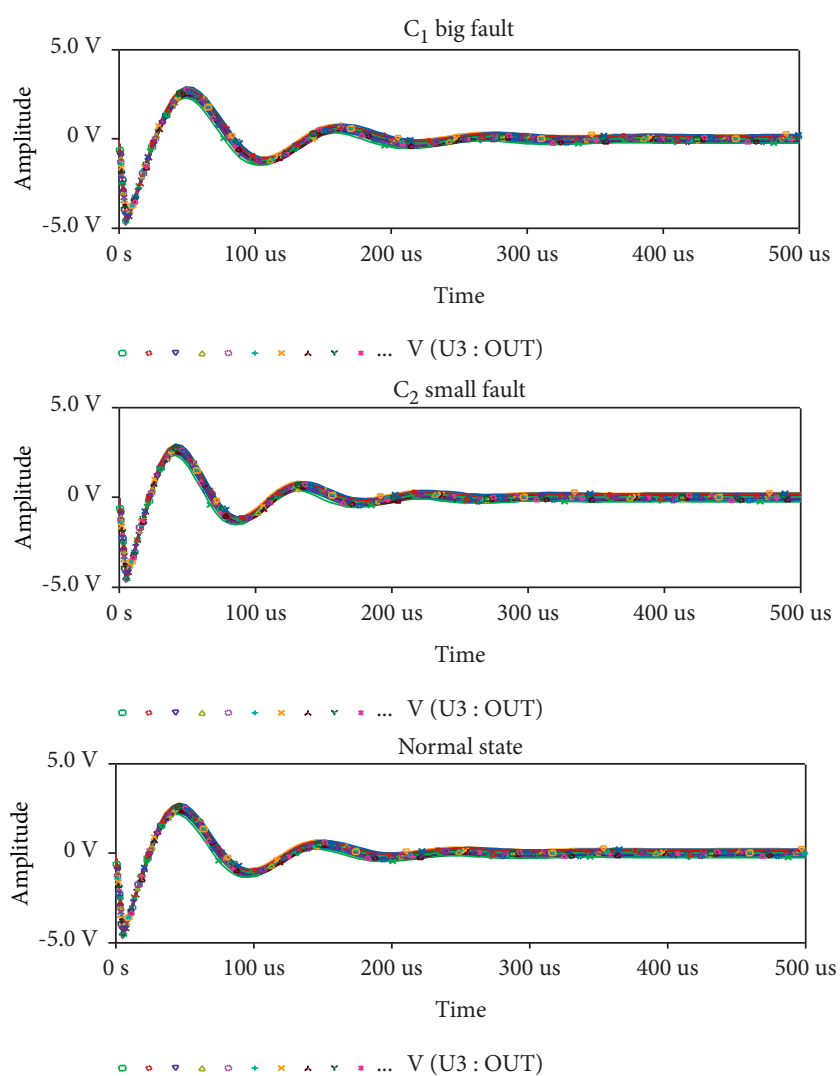

FIGURE 4: Fault response curves of four-opamp biquad high-pass filter.

Let the quadratic sum of each frequency band signal decomposed by wavelet packet be the wavelet packet energy spectrum. Assume $d_{i, j}(k)$ denotes the signal decomposed by wavelet packet. The energy of the signal in each frequency band is given by

$$
E_{i, j}=\sum_{K=1}^{N}\left|d_{i, j}(k)\right|^{2}, \quad j=0,1, \cdots 2^{i-1},
$$

where $N$ denotes the length of original signal. Let the wavelet packet energy spectrum be $E$, which is constituted of all $E_{i, j}$ as follows:

$$
E=\left[E_{i, 0}, E_{i, 1}, \cdots E_{i, j}, \cdots, E_{i, 2^{i}-1}\right] .
$$

When the energy is large, it can be normalized as follows:

$$
\sum E=E_{i, 0}+E_{i, 1}+\cdots+E_{i, j}+\cdots+E_{i, 2^{i}-1} .
$$

The feature vector is defined as follows:

$$
E^{\prime}=\frac{E}{\sum E} \text {. }
$$

50 samples are collected for each fault model, and each sample has 8 attributes. Partial fault features of the wavelet packet energy spectrum are shown as Figure 6, where $x$-axis represents eight bands by three-layer wavelet packet decomposition, $y$-axis represents analog circuit fault category, and $z$-axis represents energy value by wavelet packet decomposition.
In order to reduce redundancy and improve the recognition rate of analog circuit fault diagnosis, the sparse random projections algorithm mentioned above is used to further extract the fault features of analog circuit. Figure 7 is a three-dimensional fault information distribution map extracted with sparse random projections.

Each fault feature is clearly divided in Figure 7. It shows that the sparse random projections feature extraction algorithm can reflect the essence of analog circuit faults and has better clustering and distinguishing ability for analog circuit faults, which creates a good condition for subsequent fault diagnosis.

In order to diagnosis circuit fault through KNN classifier, the extracted fault features are divided into two data sets. 30 samples are used as training data, and the rest 20 sample are test data. The total training data and test data are 390 and 260, respectively.

Table 2 lists diagnosis results of four-opamp biquad high-pass filter circuit using different classifiers. From $\mathrm{Ta}$ ble 2, the proposed method diagnoses all kinds of faults correctly. It achieves $100 \%$ classification. The accuracy rate of RBF classifier is $65.77 \%$, and the fuzzy fault number is 8 . The result of SVM is $81.92 \%$. Its fuzzy fault number is 9 . The accuracy rate of BayesNet and decision tree is $97.31 \%$ and $96.54 \%$, respectively, and the fuzzy fault number is 4 and 5 , respectively. Obviously, the proposed method is better than other methods.

Fuzzy fault sets are listed in Table 3. The SRP-KNN method obtains the least fuzzy faults. RBF classifier cannot 

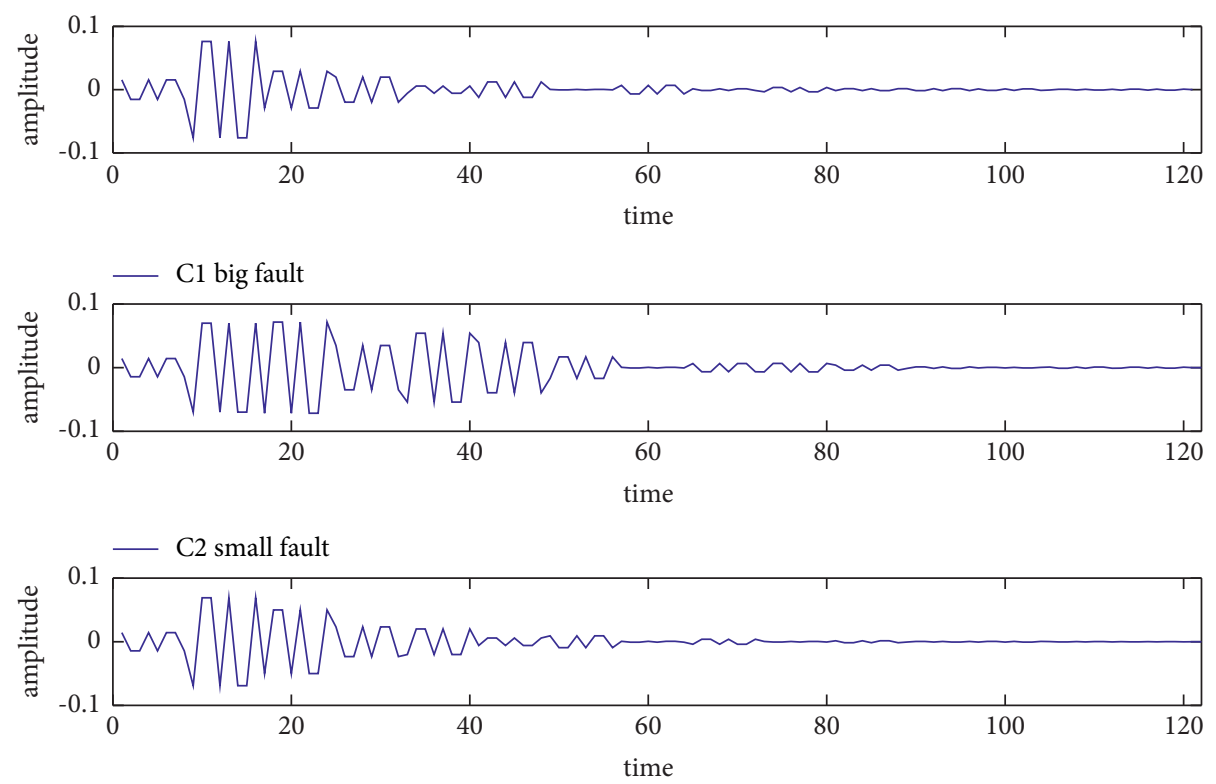

- normal state

FIgURE 5: Fault curves of 3-layer Haar wavelet packet decomposition.

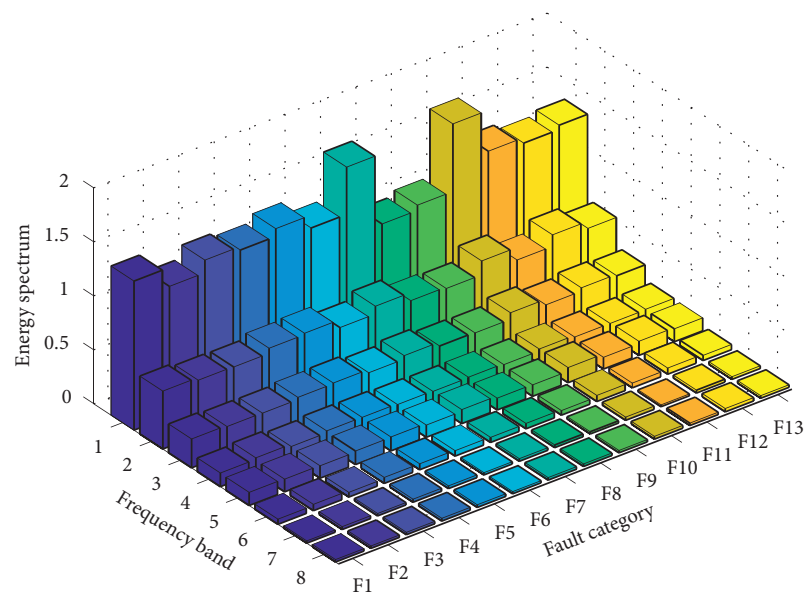

Figure 6: Partial wavelet packet energy spectrum fault features.

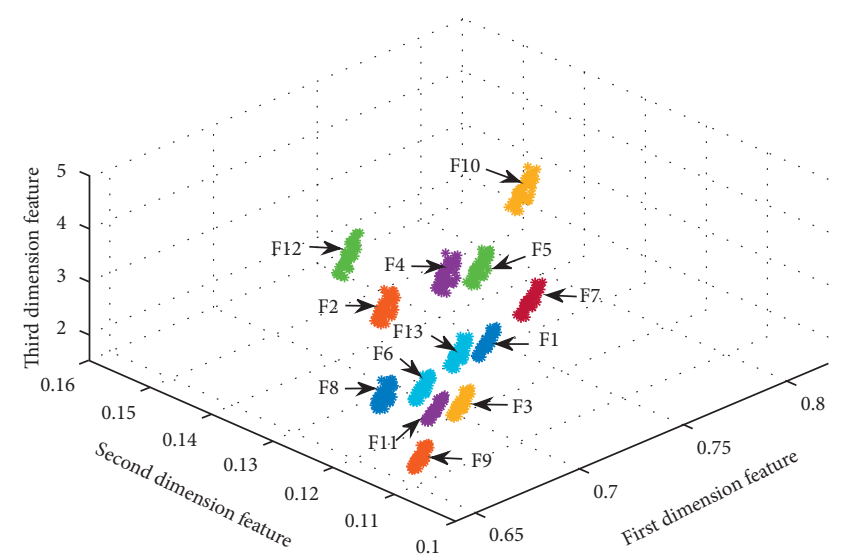

FIGURE 7: Scatter plot of the 3D fault feature extracted with SRP.

TABLE 2: Diagnosis results of four-opamp biquad high-pass filter circuit.

\begin{tabular}{|c|c|c|c|c|c|}
\hline Fault code & The proposed method (\%) & RBFclassifier (\%) & BayesNet (\%) & Decision tree $(\%)$ & SVM (\%) \\
\hline$F 1$ & 100 & 35 & 100 & 90 & 100 \\
\hline$F 2$ & 100 & 100 & 100 & 100 & 90 \\
\hline F3 & 100 & 100 & 85 & 100 & 55 \\
\hline F4 & 100 & 10 & 90 & 90 & 70 \\
\hline F5 & 100 & 0 & 100 & 100 & 90 \\
\hline F6 & 100 & 95 & 100 & 100 & 90 \\
\hline F7 & 100 & 70 & 100 & 100 & 90 \\
\hline F8 & 100 & 55 & 100 & 100 & 100 \\
\hline F9 & 100 & 100 & 100 & 95 & 100 \\
\hline$F 10$ & 100 & 100 & 100 & 100 & 100 \\
\hline$F 11$ & 100 & 0 & 100 & 90 & 50 \\
\hline$F 12$ & 100 & 100 & 95 & 100 & 65 \\
\hline$F 13$ & 100 & 90 & 95 & 90 & 65 \\
\hline Average result & 100 & 65.77 & 97.31 & 96.54 & 81.92 \\
\hline
\end{tabular}


TABLE 3: Fuzzy fault number for various diagnosis methods.

\begin{tabular}{lcc}
\hline Method & Fuzzy fault number & Fuzzy fault \\
\hline RBF classifier & 8 & $F 1, F 4-F 8, F 11, F 13$ \\
BayesNet & 4 & $F 3, F 4, F 12, F 13$ \\
Decision tree & 5 & $F 1, F 4, F 9, F 11, F 13$ \\
SVM & 9 & $F 2-F 7, F 11-F 13$ \\
The proposed method & 0 & - \\
\hline
\end{tabular}

Table 4: Diagnosis results in the noisy environment.

\begin{tabular}{|c|c|c|c|c|}
\hline Fault code & The proposed method (\%) & BayesNet (\%) & Decision tree $(\%)$ & SVM (\%) \\
\hline$F 1$ & 100 & 99.5 & 99.5 & 93 \\
\hline$F 2$ & 98 & 94 & 92.5 & 80 \\
\hline F3 & 99 & 90 & 96.5 & 54.5 \\
\hline$F 4$ & 100 & 96.5 & 95 & 60.5 \\
\hline F5 & 100 & 93.5 & 96 & 76 \\
\hline F6 & 100 & 95 & 95 & 78 \\
\hline F7 & 100 & 100 & 99.5 & 77 \\
\hline$F 8$ & 100 & 97.5 & 99 & 94 \\
\hline$F 9$ & 100 & 100 & 94.5 & 97.5 \\
\hline$F 10$ & 100 & 100 & 99.5 & 92.5 \\
\hline$F 11$ & 98 & 96 & 93.5 & 57 \\
\hline$F 12$ & 99.5 & 92.5 & 96 & 65.5 \\
\hline$F 13$ & 99 & 96 & 95 & 57.5 \\
\hline Average result & 99.5 & 96.19 & 96.27 & 75.62 \\
\hline
\end{tabular}

locate fuzzy fault set of $\{F 2, F 4, F 5, F 6, F 7, F 8, F 11, F 13\}$. SVM cannot locate fuzzy fault set of $\{F 2, F 3, F 4, F 5, F 6, F 7$, $F 11, F 12, F 13\}$. Fuzzy fault set of $\{F 3, F 4, F 12, F 13\}$ and $\{F 1$, $F 4, F 9, F 11, F 13$ \} cannot be correctly diagnosed for the methods in BayesNet and decision tree. These show that the proposed method obtains a decent performance for fouropamp biquad high-pass filter.

In order to further illustrate the superiority of the proposed method, white noise is added to the fault information. In order to reflect the randomness of noise, the experiment is repeated ten times, and the average value of the ten experiments is recorded as the final diagnosis result. Table 4 lists diagnosis results in the noisy environment.

From Table 4, the accuracy of fault diagnosis has decreased slightly in the noisy environment. This shows that noise can affect the results of analog circuit fault diagnosis. The accuracy rate of the proposed method is $99.5 \%$. The accuracy rate of BayesNet and decision tree is $96.19 \%$ and $96.27 \%$, respectively. The accuracy rate of SVM is only $75.62 \%$, which is much lower. The proposed method can diagnose eight fault modes, and BayesNet can only diagnose three fault modes. None of the fault modes can be completely diagnosed correctly by decision tree and SVM. It can be seen that SVM performs the worst in the noisy environment.

Fault diagnosis accuracy rate curves of ten experiments in noisy environment are drawn in Figure 8. From Figure 8, the accuracy rate of the proposed method is always the highest, and it is $100 \%$ in seven experiments. The accuracy rate of BayesNet, decision tree, and SVM is significantly lower than that of the proposed method. In addition, the accuracy rate of BayesNet, decision tree, and SVM fluctuates greatly. It indicates that these methods are more sensitive to

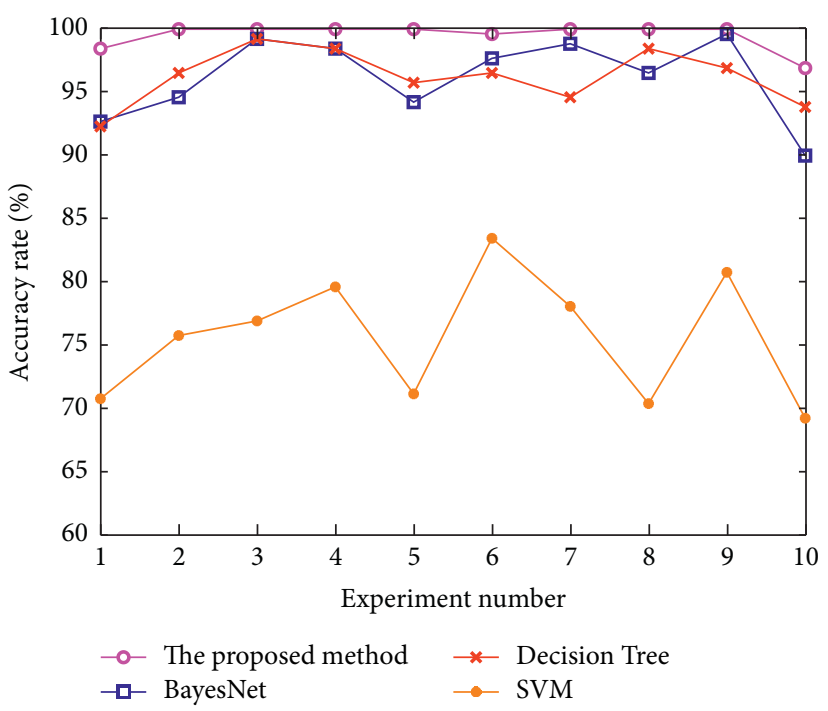

FIGURE 8: Fault diagnosis accuracy curves in noisy environment.

noise. Therefore, the proposed method is significantly better than BayesNet, decision tree, and SVM in noisy environment. In summary, the fault diagnosis results prove that the introduced method can more effectively diagnose soft faults for analog circuit.

\section{Conclusion}

In this paper, sparse random projections and $\mathrm{K}$-nearest neighbor classifier techniques are applied for fault diagnosis in analog circuit. The study indicates that the preprocessing technique has a significant impact on fault diagnosis of 
analog circuit. The proposed method uses the wavelet packet energy spectrum and sparse random projections as preprocessor to extract analog circuit fault feature. It is contributed to reduce noise interference and test cost in fault diagnosis, and dimensionality reduction using SPR can lead to more effective KNN classification. Experiment results show that the introduced SRP-KNN-based method performs better than existing methods for analog circuit fault diagnosis even when noise is added. According analog circuit theory, the response of the nonlinear system is different from that of the linear system. Hence, the proposed method will be studied to diagnose nonlinear circuit fault in our further research.

\section{Data Availability}

The data used to support the findings of this study are available from the author upon request.

\section{Conflicts of Interest}

The authors declare that there are no conflicts of interest.

\section{Acknowledgments}

This work was supported in part by Research Foundation of the Jinling Institute of Technology for Advanced Talents under Grant jit-b-201631 and in part by the Natural Science Foundation of Jiangsu Province under Grant BK20161104.

\section{References}

[1] C. Alippi, M. Catelani, A. Fort, and M. Mugnaini, "SBT soft fault diagnosis in analog electronic circuits: a sensitivity-based approach by randomized algorithms," IEEE Transactions on Instrumentation and Measurement, vol. 51, no. 5, pp. 11161125, 2002.

[2] W. G. Fenton, T. M. Mcginnity, and L. P. Maguire, "Fault diagnosis of electronic systems using intelligent techniques: a review," IEEE Transactions on Systems, Man and Cybernetics, Part C (Applications and Reviews), vol. 31, no. 3, pp. 269-281, 2001.

[3] A. Sai Sarathi Vasan, B. Long, and M. Pecht, "Diagnostics and prognostics method for analog electronic circuits," IEEE Transactions on Industrial Electronics, vol. 60, no. 11, pp. 5277-5291, 2013.

[4] C. Yang, "Multiple soft fault diagnosis of analog filter circuit based on genetic algorithm," IEEE Access, vol. 8, pp. 81938201, Jan. 2020.

[5] W. He, Y. He, and B. Li, "Generative adversarial networks with comprehensive wavelet feature for fault diagnosis of analog circuits," IEEE Transactions on Instrumentation and Measurement, vol. 69, no. 9, pp. 6640-6650, Sept. 2020.

[6] F. Aminian, M. Aminian, and H. W. Collins, "Analog fault diagnosis of actual circuits using neural networks," IEEE Transactions on Instrumentation and Measurement, vol. 51, no. 3, pp. 544-550, 2002.

[7] C. Yang, S. Tian, B. Long, and F Chen, "Methods of handling the tolerance and test-point selection problem for analogcircuit fault diagnosis," IEEE Transactions on Instrumentation and Measurement, vol. 60, no. 1, pp. 176-185, 2010.
[8] Z. Yuan, Y. He, L. Yuan, and Z Cheng, “A diagnostics method for analog circuits based on improved kernel entropy component analysis," Journal of Electronic Testing, vol. 33, no. 2, pp. 697-707, 2017.

[9] H. Han, H. Wang, S. Tian, and N. Zhang, "A new analog circuit fault diagnosis method based on improved mahalanobis distance," Journal of Electronic Testing, vol. 29, no. 1, pp. 95-102, 2013.

[10] Q. Ma, Y. He, and F. Zhou, "A new decision tree approach of support vector machine for analog circuit fault diagnosis," Analog Integrated Circuits and Signal Processing, vol. 88, no. 3, pp. 455-463, 2016.

[11] H. E. Yigang, L. I. Chenchen, T. Wang, T. Shi, L. Tao, and W. Yuan, "Incipient fault diagnosis method for IGBT drive circuit based on improved SAE," IEEE Access, vol. 7, pp. 92410-92418, 2019.

[12] W. Yu, Y. Sui, and J. Wang, "The faults diagnostic analysis for analog circuit based on FA-TM-ELM," Journal of Electronic Testing, vol. 32, no. 4, pp. 459-465, 2016.

[13] M. Sheikhan and A. A. Sha'bani, "PSO-optimized modular neural network trained by OWO-HWO algorithm for fault location in analog circuits," Neural Computing \& Applications, vol. 23, no. 2, pp. 519-530, 2013.

[14] Z. Yuan, Y. He, and L. Yuan, “An efficient feature extraction approach based on manifold learning for analogue circuits fault diagnosis," Analog Integrated Circuits and Signal Processing, vol. 102, no. 1, pp. 237-252, 2018.

[15] F. Aminian and M. Aminian, "fault diagnosis of nonlinear analog circuits using neural networks with wavelet and fourier transforms as preprocessors," Journal of Electronic Testing, vol. 17, no. 6, pp. 471-481, 2001.

[16] P. Song, Y. He, and W. Cui, "Statistical property feature extraction based on FRFT for fault diagnosis of analog circuits," Analog Integrated Circuits and Signal Processing, vol. 87, no. 3, pp. 427-436, 2016.

[17] C. Zhang, Y. He, L. Yuan, and S. Xiang, "Analog circuit incipient fault diagnosis method using DBN based features extraction," IEEE Access, vol. 6, pp. 23053-23064, 2018.

[18] H. Luo, W. Lu, and Y. Wang, "A novel approach for analog fault diagnosis based on stochastic signal analysis and improved GHMM," Measurement, vol. 81, pp. 26-35, 2016.

[19] Y. Xiao and L. Feng, "A novel linear ridgelet network approach for analog fault diagnosis using wavelet-based fractal analysis and kernel PCA as preprocessors," Measurement, vol. 45, no. 3, pp. 297-310, 2012.

[20] Y. Xiao, L. Feng, and Y. He, "A fault diagnosis approach of analog circuit using wavelet-based fractal analysis and kernel LDA," Transactions of China Electrotechnical Society, vol. 27, no. 8, pp. 230-238, 2012.

[21] F. Aminian and M. Aminian, "fault diagnosis of analog circuits using bayesian neural networks with wavelet transform as preprocessor," Journal of Electronic Testing, vol. 17, no. 1, pp. 29-36, 2001.

[22] H. Luo, Y. W. Jiang Cui, and C. Jiang, "A SVDD approach of fuzzy classification for analog circuit fault diagnosis with FWT as preprocessor," Expert Systems with Applications, vol. 38, no. 8, pp. 10554-10561, 2011.

[23] W. He, Y. He, B. Li, and C. Zhang, "A naive-bayes-based fault diagnosis approach for analog circuit by using image-oriented feature extraction and selection technique," IEEE Access, vol. 8, pp. 5065-5079, 2019.

[24] X. Yuan, Z. Liu, Z. Miao, Z. Zhao, F. Zhou, and Y. Song, "fault diagnosis of analog circuits based on IH-PSO optimized 
support vector machine," IEEE Access, vol. 7, pp. 137945137958, 2019.

[25] C. Olivier, V. Vapnik, O. Bousquet, and S. Mukherjee, "Choosing multiple parameters for support vector machines," Machine Learning, vol. 46, pp. 131-159, 2002.

[26] S. Zhang, X. Li, Z. Ming, X. Zhu, and D. Cheng, "Learning k for kNN Classification," ACM Transactions on Intelligent Systems and Technology, vol. 8, no. 8, pp. 1-19, 2017.

[27] Z. Zhe Zhou, C. Chunjie Yang, and C. Yang, "fault detection using random projections and $\mathrm{k}$-nearest neighbor rule for semiconductor manufacturing processes," IEEE Transactions on Semiconductor Manufacturing, vol. 28, no. 1, pp. 70-79, 2015.

[28] J. E. Fowler and D. Qian, "Anomaly detection and reconstruction from random projections," IEEE Transactions on Image Processing, vol. 21, no. 1, pp. 84-95, 2012.

[29] P. W. Fieguth and D. Dewen Hu, "Fusing sorted random projections for robust texture and material classification," IEEE Transactions on Circuits and Systems for Video Technology, vol. 25, no. 3, pp. 482-496, 2015.

[30] J. Haupt and R. Nowak, "Signal reconstruction from noisy random projections," IEEE Transactions on Information Theory, vol. 52, no. 9, pp. 4036-4048, 2006.

[31] E. Bingham and H. Mannila, "Random projection in dimensionality reduction: applications to image and text data," in Proceedings of the Seventh ACM SIGKDD International Conference on Knowledge Discovery and Date Mining, pp. 245-250, ACM SIGKDD, San Francisco, California, USA, 26 August2001.

[32] M. A. Cheema, W. Zhang, and X. Lin, "Continuous reverse k nearest neighbors queries in Euclidean space and in spatial networks," The VLDB Journal, vol. 21, no. 1, pp. 69-95, 2012. 